\title{
Clinical application of chromosome 9p21.3 genotyping in patients with coronary artery disease
}

\author{
SVETLANA NIKULINA $^{1}$, IVAN ARTYUKHOV ${ }^{1}$, PAVEL SHESTERNYA ${ }^{1}$, OKSANA GAVRILYUK ${ }^{1}$, \\ VLADIMIR MAKSIMOV $^{2}$, MIKHAIL VOYEVODA ${ }^{2}$ and DENIS BRUSENTSOV ${ }^{1}$ \\ ${ }^{1}$ Department of Internal Diseases No. 1, Professor V.F. Voino-Yasenetsky Krasnoyarsk State \\ Medical University of The Ministry of Healthcare of The Russian Federation, Krasnoyarsk 660022; \\ ${ }^{2}$ Laboratory of Molecular Genetic Studies of Therapeutic Diseases, Institute of Internal and Preventive Medicine of \\ The Siberian Branch of The Russian Academy of Medical Sciences, Novosibirsk 630089, Russian Federation
}

Received January 27, 2018; Accepted October 3, 2018

DOI: $10.3892 /$ etm.2019.7884

\begin{abstract}
The aim of the present study was to investigate the susceptibility of two coronary artery disease (CAD)-associated single nucleotide polymorphisms on 9p21 (rs1333049 and rs 10757278) to myocardial infarction (MI) in a primary (stratification of high risk group for MI) and secondary prevention setting. The prospective observational study included 500 patients with MI [411 males (82.2\%) and 89 females $(17.8 \%)]$ under 65 years. The risk of MI for carriers of the homozygous CC genotype of $r s 1333049$ and homozygous GG genotype of $r s 10757278$ was 1.77 [95\% confidence interval (CI): 1.36-2.37], and 1.70 (95\% CI: 1.24-2.32) respectively. The risk of MI for heterozygous allele carriers was slightly lower. Specifically, the risk of MI was 1.58 (95\% CI: 1.18-2.11) for both heterozygous and homozygous carriers of the rs 1333049 $\mathrm{C}$ allele, and 1.36 (95\% CI: 1.01-1.83) for the carriers of the rs $10757278 \mathrm{G}$ allele. A logistic regression model including sex, age, presence of excess weight or obesity, abdominal obesity, diabetes mellitus, arterial hypertension, hypercholesterolemia, positive family history and smoking status parameters revealed that $r s 1333049 \mathrm{CC}$ genotype was an independent predictive factor of myocardial infraction [OR=1.71 (95\% CI: 1.16-2.52), $\mathrm{P}=0.006]$. Patients who underwent percutaneous coronary intervention (PCI) during index hospitalization and patients who did not receive PCI were followed up for two years after discharge. Compared with patients with MI who underwent PCI, the risk of recurrent acute coronary syndrome (re-ACS)
\end{abstract}

Correspondence to: Mrs. Oksana Gavrilyuk, Department of Internal Diseases No. 1, Professor V.F. Voino-Yasenetsky Krasnoyarsk State Medical University of The Ministry of Healthcare of The Russian Federation, 1 Partizan Zheleznyak Street, Krasnoyarsk 660022, Russian Federation

E-mail: oksana.gavrilyuk@mail.ru

Key words: cardiogenetics, clinical application of genomic data, myocardial infarction, percutaneous coronary intervention, prevention was higher among $r s 1333049 \mathrm{C}$ allele carriers who did not receive PCI during index hospitalization. One year after MI, the OR of re-ACS was 4.91 (95\% CI: 1.45-16.66), while two years after MI, OR was 3.77 (95\% CI: 1.50-9.52) in those patients who did not receive PCI during index hospitalization. There was no statistically significant association between polymorphic variants of $r s 1333049$ and MI follow-up outcomes in patients who underwent PCI. The present study indicated clinical utility of 9p21.3 genotyping to predict the outcomes for patients with MI without PCI. Due to the small sample size, this association study forms basis for larger, nationwide studies investigating clinical applications of genetic data.

\section{Introduction}

High incidence of both ST-elevation (STEMI) and non-ST-elevation acute myocardial infarction (NSTEMI) reported in published genetic research (1) determines the necessity of studying genetic aspects that can contribute to the development of primary and secondary myocardial infarction (MI) prevention programs.

The association between the 9p21.3 locus and coronary artery disease (CAD), and MI has become one of the important discoveries of modern cardiogenetics $(1,2)$. The association between this genome locus and coronary atherosclerosis, and MI has been demonstrated in various populations (3-8). However, the underlying mechanism remains to be elucidated. It has been demonstrated that a non-coding RNA of locus 9p21.3 affected cardiac expression of cyclin dependent kinase inhibitor $2 \mathrm{~A}$ and $\mathrm{B}$ genes, and may lead to a lower index of atherosclerotic plaque stability (9). Further studies are necessary to enable the clinical implementation of this emerging marker in risk stratification and outcome of patients with cardiovascular diseases. To assess the utility of genetic markers that may reflect the risk of MI, the existing risk calculator and the Net Reclassification Improvement (NRI) method are usually used. Previously, results of several large-scale projects on the integration of genomic information into the existing CAD and MI risk rating scales have been published, however, the results were inconsistent (10-12). 
By contrast, the clinical utility of 9p21.3 locus genotyping to predict the outcomes for patients following MI may be more promising compared with other loci, since it was replicated as a genetic predictor in almost all ethnic groups and races $(3,13)$. In particular, for patients with acute coronary syndrome (ACS) enrolled in the Global Registry of Acute Coronary Events (GRACE), Buysschaert et al (13) reported the results of inclusion of the 9p21.3 locus genotyping data into the GRACE risk score. A positive association between the 9p21.3 locus and recurrent MI or cardiac death within the first 6 months after the primary event was revealed (13). Adrissino et al (14) proposed the use of risk allele C (SNP rs1333040) in secondary MI prevention in people under 45 years.

Significant associations have been revealed between the risk 9p21.3 genotypes and the outcomes in the most severe cases of MI with multi-vessel coronary artery burden or high risk according to the GRACE score $(15,16)$. The results of the present study indicated that future clinical application of genomic data requires accurate definition of the target group of patients. Since percutaneous coronary intervention (PCI) can markedly alter the prognosis of patients with $\mathrm{MI}$, the present study aimed to estimate the risk of MI among patients with different SNPs at 9p21.3 receiving differential hospital-based treatment.

\section{Materials and methods}

Patients. The participants of the present study were patients admitted to the two largest cardiological hospitals in Krasnoyarsk, Russian Federation: Regional Clinical Hospital and Krasnoyarsk Interdistrict Clinical Hospital Number 20 named after I.S. Berzon. The inclusion criteria compromised: i) Confirmed diagnosis of MI verified by electrocardiogram (ECG) and such clinical symptoms and cardiac markers, including typical anginal pain lasting more than 20 min or atypical manifestations such as abdominal epigastric pain, indigestion-like symptoms and isolated dyspnoea; ii) age under 65 years, iii) Caucasian origin; and iv) signed informed consent. The present study was approved by the Ethics Committee of Professor V.F. Voino-Yasenetsky Krasnoyarsk State Medical University.

A total of 500 patients with MI [22-65 years; median age, 54.0; lower quartile $\left(\mathrm{Q}_{25}\right)$, 48.0; upper quartile $\left(\mathrm{Q}_{75}\right), 59.0$ ] were included in the present study conducted from January 1, 2009 to the June 30, 2010. The group of patients with MI included 411 males $(82.2 \%)$ and 89 females $(17.8 \%)$. The results of 31 autopsies [24 males and 7 females, 44-65 years (57.87 \pm 5.92 years)] with confirmed diagnosis of MI as the cause of out-of-hospital sudden death during the same period of time were also included in the present study.

The control group subjects were selected at random from the Novosibirsk samples of two epidemiological studies: Multinational Monitoring of Trends and Determinants in Cardiovascular Disease (17), and Health, Alcohol and Psychosocial factors In Eastern Europe (HAPIEE) (18), conducted at the Novosibirsk Institute of Internal and Preventive Medicine (Russian Federation) to investigate angina and its equivalents with the use of cardiospecific markers (troponin, creatine phosphokinase-MB fraction). Based on the results of the Rose Angina Questionnaire, a total of 535 individuals without CAD were selected among the HAPIEE participants enrolled from Novosibirsk. The control group included 423 $(79.1 \%)$ males and $112(20.9 \%)$ females $\leq 65$ years (median, 55.0; $\left.\mathrm{Q}_{25}, 48.0 ; \mathrm{Q}_{75}, 61.0\right)$.

The two groups did not exhibit any significant differences in the mean age $(\mathrm{P}=0.351)$ or in the presence of obesity (including abdominal obesity) and excess weight, as presented in Table I. In the MI group, a statistically significant predominance of smokers, arterial hypertension, diabetes mellitus and positive family history were identified. However, hypercholesterolemia was significantly more frequent in the control group. This can be due to a widespread use of statins in the treatment of MI. Hypercholesteremia was diagnosed if total cholesterol level was $>5 \mathrm{mmol} / \mathrm{l}$. The World Health Organization classification based on body mass index (BMI) calculation using the following equation: $\mathrm{BMI}=$ weight in kilograms/height in meters ${ }^{2}$, was used to diagnose obesity and excess weight. Abdominal obesity was diagnosed in the case of waist circumference of $\geq 80 \mathrm{~cm}$ in women and $\geq 94 \mathrm{~cm}$ in men. The presence of MI, angina pectoris or sudden cardiac death in parents were considered as positive family history. Sex differences in the described risk factors between the study group and the control group are presented in Table II.

A total of $14.5 \%$ of the study patients exhibited NSTEMI, while the majority of the study participants $(85.5 \%)$ exhibited STEMI. ECG results revealed the presence of pathological Q-waves in two thirds of patients. The cases of anterior and anterior lateral MI were assigned to the anterior MI localization, while the cases of inferior, inferior lateral and posterior MI were assigned to the non-anterior MI localization.

The described categories of MI cases are presented in Table III. The results are available for 496 patients with MI as 3 patients exhibited left bundle branch block and 1 patient had an implanted cardiostimulator. The severity and risk of unfavorable outcomes were assessed in each hospitalized study patient using the Global Registry of Acute Coronary Events (GRACE) (19) and Thrombolysis in Myocardial Infarction (TIMI) risk scores (20). Interventional reperfusion was performed in 251 (50.2\%) of 500 patients with MI. In 232 patients $(92.4 \%)$ PCI involved coronary stent insertion. A total of 249 patients $(49.8 \%)$ received conservative treatment with antithrombotics, anticoagulants, nitrates, $\beta$-blockers and statins, including 88 patients $(35.3 \%)$ who received thrombolytic therapy.

Prospective medium-term follow-up period lasted two years. Patients were monitored three times at 6,12 and 24 months following discharge from the hospital. The following endpoints were assessed: Recurrent MI, hospitalization for unstable angina (UA), recurrent acute coronary syndrome (ACS) was defined as recurrent MI, hospital admission for UA and repeated PCI. A total of 476 patients were discharged from the hospital. A total of $24(5.0 \%)$ patients were lost to follow up, and, therefore, the present study analyzed the outcomes of the disease for 452 patients. To make a differential estimation of the outcomes, the discharged study participants were divided into two groups: Patients receiving the conservative therapy (group 1) and patients who underwent PCI (group 2).

All collected samples were marked and maintained in freezer storage boxes until DNA extraction. DNA extraction was performed according to the manufacturer's instructions. 
Table I. General characteristics of the study groups.

\begin{tabular}{|c|c|c|c|}
\hline Characteristic & MI, n (\%) & Control, n (\%) & P-value \\
\hline \multicolumn{4}{|l|}{ Sex } \\
\hline Male & $411(82.2)$ & $423(79.1)$ & \multirow[t]{2}{*}{0.239} \\
\hline Female & $89(17.8)$ & $112(20.9)$ & \\
\hline \multicolumn{4}{|l|}{ Weight } \\
\hline BMI 18.5-24.9 & $183(37.7)$ & $181(40.0)$ & \multirow[t]{3}{*}{0.594} \\
\hline BMI 25-29.9 & $192(39.6)$ & $181(40.0)$ & \\
\hline $\mathrm{BMI} \geq 30$ & $110(22.7)$ & $90(20.0)$ & \\
\hline \multicolumn{4}{|l|}{ Overweight and obesity } \\
\hline $\mathrm{BMI} \geq 25$ & $302(62.3)$ & $272(60.0)$ & 0.485 \\
\hline Abdominal obesity & $161(48.8)$ & $199(52.5)$ & 0.323 \\
\hline \multicolumn{4}{|l|}{ Smoking status } \\
\hline No & $150(30.9)$ & $204(38.5)$ & \multirow[t]{3}{*}{0.005} \\
\hline Yes & $305(62.9)$ & $271(51.1)$ & \\
\hline Ex-smoker & $30(6.2)$ & $55(10.4)$ & \\
\hline Arterial hypertension & 334 (68.9) & $300(56.1)$ & $<0.001$ \\
\hline Diabetes mellitus & $52(10.7)$ & $30(6.6)$ & 0.026 \\
\hline Hypercholesterolemia & $323(72.3)$ & $360(79.5)$ & 0.011 \\
\hline Positive family history & $153(33.6)$ & $107(24.4)$ & 0.002 \\
\hline
\end{tabular}

BMI, body mass index; MI, myocardial infarction.

Table II. Sex differences between the study and control groups.

\begin{tabular}{|c|c|c|c|c|c|c|}
\hline \multirow[b]{3}{*}{ Risk factors } & \multicolumn{6}{|c|}{ Sex } \\
\hline & \multicolumn{3}{|c|}{ Male } & \multicolumn{3}{|c|}{ Female } \\
\hline & MI group, n (\%) & Control group, n (\%) & P-value & MI group, n (\%) & Control group, n (\%) & P-value \\
\hline \multicolumn{7}{|l|}{ Smoking history } \\
\hline Yes & $96(23.9)$ & $111(26.4)$ & 0.007 & $54(64.3)$ & $93(85.3)$ & 0.003 \\
\hline No & $278(69.3)$ & $257(61.0)$ & & $27(32.1)$ & $14(12.8)$ & \\
\hline Ex-smoker & $27(6.7)$ & $53(12.6)$ & & $3(3.6)$ & $2(1.8)$ & \\
\hline Hypertensive disease & $272(67.8)$ & $243(57.4)$ & 0.002 & $62(73.8)$ & $57(50.9)$ & 0.001 \\
\hline Diabetes mellitus & $36(9.0)$ & $22(6.5)$ & 0.201 & $16(19.0)$ & $8(7.1)$ & 0.012 \\
\hline Positive family history & $129(34.3)$ & $80(24.2)$ & 0.003 & $24(30.4)$ & $27(24.8)$ & 0.393 \\
\hline
\end{tabular}

MI, myocardial infarction.

Specifically, DNA was extracted from whole venous blood or myocardial tissue samples using a phenol-chloroform extraction. Genotypes for the $r s 1333049$ and $r$ s10757274 SNPs were determined using the 5'nuclease assay with TaqMan probes (assay identification C__1754666_10 and C__11841860_10, respectively; Thermo Fisher Scientific, Inc., Waltham, MA, USA). The cycling conditions were $60^{\circ} \mathrm{C}$ for $2 \mathrm{~min}$ and $95^{\circ} \mathrm{C}$ for $10 \mathrm{~min}$, followed by 40 cycles of $95^{\circ} \mathrm{C}$ for $15 \mathrm{sec}$ and $60^{\circ} \mathrm{C}$ for $1 \mathrm{~min}$. Each 10- $\mu 1$ amplification reaction volume contained $1 \mathrm{X}$ Universal Master Mix (ABgene Inc., Rochester, NY, USA) and 10 ng of template DNA. Allelic discrimination was measured automatically on the ABI Prism 7900HT (Thermo Fisher Scientific, Inc) with Sequence Detection Systems 2.1 software of the Applied Biosystems (Thermo Fisher Scientific, Inc.).

Statistical analysis. Data were analyzed using the SPSS software (version 20.0; IBM Corp., Armonk, NY, USA). One sample Kolmogorov-Smirnov test was used to verify the normality of the distribution of data. The significance of differences in qualitative characteristics in the study groups was assessed using continuity-corrected nonparametric Pearson's chi-square test or Fisher's exact test when the 
Table III. Main categories of MI cases.

\begin{tabular}{lc}
\hline MI categories & $\mathrm{n}(\%)$ \\
\hline STEMI & $424(85.5)$ \\
NSTEMI & $72(14.5)$ \\
Anterior MI & $217(43.7)$ \\
Non-anterior MI & $241(48.6)$ \\
Circular MI & $38(7.7)$ \\
Q-wave & $338(68.1)$ \\
non Q-wave & $158(31.9)$ \\
\hline
\end{tabular}

$\mathrm{n}$, number of cases; MI, myocardial infarction; STEMI, ST-elevation acute myocardial infarction; NSTEMI, non-ST-elevation acute myocardial infarction.

frequency of symptom occurrence was $\leq 5$. Mann-Whitney rank test was used to assess the significance of statistical differences in quantitative characteristics. $\mathrm{P}<0.05$ was considered to indicate a statistically significant difference. To prove the statistical significance of the differences between carriers of the $\mathrm{C}$ allele and homozygous GG individuals the Wilcoxon signed-rank test and the Mantel-Cox log-rank test were used. Correlation analysis was performed using the nonparametric Spearman test. Odds ratio (OR) was calculated to assess the risk of MI for a particular allele or genotype. In case if one of the characteristics was equal to zero and it was not possible to calculate the OR, the relative risk ratios were determined for $2 \times 2$ contingency tables and confidence intervals (CI) were calculated.

\section{Results}

Association between SNPs and MI. During the first phase of the present study, the association between two SNPs or the 9p21.3 locus (rs1333049 and rs10757278) and MI were assessed in the Russian population. Analysis of genotype frequencies of the studied SNPs revealed statistically significant differences between the MI and control groups. Frequencies in the MI group compared with the control group were $28.6 \pm 2.0$ vs. $18.4 \pm 1.7 \%(\mathrm{P}<0.001)$ for the $r s 1333049$ genotype $\mathrm{CC}$ and $80.3 \pm 1.7$ vs. $72.1 \pm 2.0 \%(\mathrm{P}=0.002)$ for both heterozygous and homozygous $\mathrm{C}$ allele carriers. For the $r s 10757278$ genotype, the frequencies of GG were $27.0 \pm 2.0$ vs. $17.9 \pm 1.8 \%(\mathrm{P}=0.002)$ in the $\mathrm{MI}$ compared with the control group and $78.1 \pm 1.8$ vs. $72.4 \pm 2.1 \%(\mathrm{P}=0.041)$ for the heterozygous $\mathrm{G}$ allele carriers.

The risk of MI was 1.77 (95\% CI: 1.36-2.37) for individuals with the homozygous CC genotype of rs1333049 and 1.70 (95\% CI: 1.24-2.32) for individuals with the GG genotype of rs 10757278. For the heterozygous risk allele carriers, the OR was slightly lower than for the homozygous risk allele carriers. Specifically, the risk of MI was 1.58 (95\% CI: 1.18-2.11) for carriers of the rs $1333049 \mathrm{C}$ allele and 1.36 (95\% CI: 1.01-1.83) for the carriers of the $r s 10757278 \mathrm{G}$ allele.

Both SNPs of the 9p21.3 locus (rs1333049 and rs10757278) are sufficiently close for linkage disequilibrium at a distance of 1,025 base pairs. The correlation coefficient between the homozygous $\mathrm{CC}$ genotype at $r s 1333049$ and the GG genotype at $r s 10757278$ was equal to 0.943 ; and 0.954 between the heterozygous genotype carriers. Consequently, these SNPs in the study population may belong to the same linkage group. There was a direct positive correlation between MI and homozygous risk genotypes of both SNPs, though absolute correlation coefficient was moderate for both the $r$ s1333049 $\mathrm{CC}$ genotype $\left(\mathrm{r}_{\mathrm{s}}=0.120\right)$ and the $r s 10757278 \mathrm{GG}$ genotype $\left(r_{\mathrm{s}}=0.108\right)$.

Regression analysis. Among the 1,035 study participants (patients with MI and control subjects), 628 individuals $(60.7 \%)$ with complete baseline information were selected for regression analysis. The MI status was presented as a dichotomous variable, where 0 indicated the absence of MI and 1 the presence of MI. The genotyping results and all risk factors evaluated in the present study, including sex, age, presence of excess weight or obesity, abdominal obesity, diabetes mellitus, arterial hypertension, hypercholesterolemia, positive family history and smoking status were included into the logistic regression analysis. Logistic regression model was built using step-by-step inclusion of prognostic factors and determination of the minimum set of predictors by calculating Nagelkerke's $\mathrm{R}$ squared values $\left(\mathrm{R}^{2}\right)$ to indicate the effect of all model predictors on response variable dispersion.

Variables with low significance and autocorrelation were rejected. The adjusted coefficient of determination $\mathrm{R}^{2}$ of a combination of variables including age, smoking status, presence of arterial hypertension, diabetes mellitus and positive family history was $0.284(\mathrm{P}<0.001)$. Among the included determinants, the CC genotype of rs1333049 was statistically significant. Following the addition of rs1333049 to the logistic regression model, adjusted $\mathrm{R}^{2}$ increased to $0.297(\mathrm{P}=0.006)$. In the logistic regression model, the $r s 1333049$ CC genotype exhibited an independent predictive value $(\mathrm{OR}=1.71 ; 95 \% \mathrm{CI}$ : 1.16-2.52; $\mathrm{P}=0.006)$.

The association between the $r s 1333049$ genotypes and the GRACE risk score was also assessed. The CC genotype of rs 1333049 was associated with intermediate and high GRACE risk scores in patients with STEMI. A total of $20.4 \pm 2.7 \%$ STEMI carriers were in the low-risk group, $32.3 \pm 4.2 \%$ in the intermediate-risk group and $28.6 \pm 7.6 \%$ in the high-risk group. There was no association between the GRACE risk scores and rs1333049 genotypes in patients with NSTEMI. Furthermore, there was no association between the rs1333049 genotypes and the TIMI score in patients with NSTEMI.

Associations between risk genotypes and patient outcomes. The second stage of the present study was to determine the association between risk genotypes and medium-term outcomes in patients with MI. Within the two-year follow-up period after discharge from the hospital, $36.5 \%$ of patients with MI were re-admitted with ACS, $13.7 \%$ of patients exhibited recurrent $\mathrm{MI}$ and $25.0 \%$ of patients underwent a new coronary intervention. Mortality among the discharged patients with MI was $2.2,3.3$ and $6.2 \%$ within 6,12 , and 24 months of the observation, respectively. Cumulative frequencies of the endpoints during the two-year observation period are presented in Fig. 1.

During the observation period, the cumulative ACS frequency was significantly higher among the carriers of the 


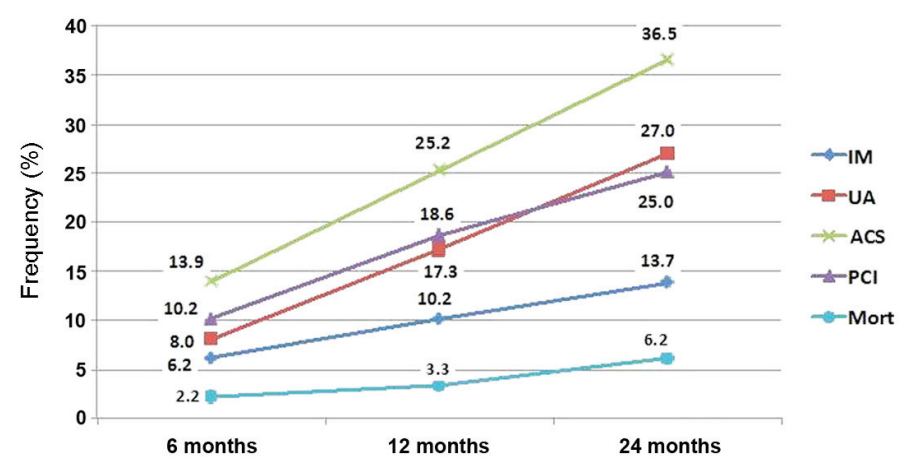

Figure 1. Medium-term outcomes of patients with MI observed during two years of follow-up. Mort, mortality; MI, myocardial infraction; UA, unstable angina; ACS, acute coronary syndrome; PCI, percutaneous coronary intervention.

$\mathrm{CC}$ and $\mathrm{CG}$ genotypes compared with patients with homozygous GG rs 1333049 genotype. At 6 months, the cumulative ACS frequency was $16.5 \pm 2.1 \%$ among carriers of the $\mathrm{CC}$ and CG genotypes compared with $4.7 \pm 2.3 \%$ among carriers of the homozygous GG genotype of $r s 1333049(\mathrm{P}=0.005)$. The difference between $\mathrm{C}$ allele carriers and $\mathrm{GG}$ homozygotes was $27.8 \pm 2.5$ vs. $16.3 \pm 4.0 \%(\mathrm{P}=0.028)$ at 12 months and $41.1 \pm 2.7 \%$ vs. $21.4 \pm 4.4 \%(\mathrm{P}=0.001)$ at 24 months, respectively (Fig. 2).

Since the polymorphism at the 9p21.3 locus is known to be associated with the severity of atherosclerotic coronary lesions, it was hypothesized that the association between genetic predictors and outcomes of MI could differ between groups of patients receiving PCI and patients receiving non-interventional procedures. In such a way, according to our hypothesis, data on genotype variations can be used in clinical practice.

Analysis of the medium-term outcomes of MI in different rs1333049 genotype carrier was performed. Patients receiving the conservative therapy (group 1) and patients who underwent PCI (group 2) exhibited no statistically significant differences in the frequency of $r s 1333049$ polymorphic variants. Thus, in group 1, there were $31.8 \pm 3.0 \%$ of the $\mathrm{CC}$ genotype carriers, and in group $2-27.0 \pm 2.9 \%(\mathrm{P}=0.239) ; 79.2 \pm 2.6$ and $81.3 \pm 2.5 \%$ of patients in groups 1 and 2, respectively, were $\mathrm{C}$ allele carriers $(\mathrm{P}=0.553)$. In addition, the groups did not differ in the total number of endpoints during the follow up period. The frequency of recurrent ACS in group 2 compared with group 1 was $12.7 \pm 2.2$ and $15.2 \pm 2.4 \%(\mathrm{P}=0.428)$ during the 6 months after MI; $24.0 \pm 2.8$ and 26. $\pm 3.0 \%(\mathrm{P}=0.550)$ within 12 months; and $35.7 \pm 3.2$ and $38.4 \pm 3.3 \%(\mathrm{P}=0.559)$ within two years of the follow up period, respectively.

Having received conservative therapy rather than PCI, group 1 had a statistically significant association of the $\mathrm{C}$ allele at rs1333049 with recurrent ACS and subsequent PCI (Fig. 3). The probability of recurrent ACS in group 1 was 4.91 (95\% CI: 1.45-16.66) a year after MI and 3.77 (95\% CI: 1.50-9.52) after two years. No statistically significant association between the rs1333049 polymorphic variants and MI outcomes was revealed in the medium-term follow up of patients who underwent PCI (Fig. 4). The frequency of recurrent MI and hospitalization for ACS did not differ significantly within a year after MI between the carriers of the $\mathrm{C}$ allele and individuals with homozygous GG genotype of $r s 1333049$ [11.4 \pm 2.4 vs. $11.6 \pm 4.9 \%(\mathrm{P}=0.961)$ and $24.4 \pm 3.2$ vs. $25.6 \pm 6.7 \%(\mathrm{P}=0.875)$, respectively]. During the two-year observation period, the frequency of recurrent MI (15.4 $\pm 2.7 \%)$ and ACS $(37.7 \pm 3.7 \%)$ was higher in carriers of the $r s 1333049 \mathrm{C}$ risk allele compared with the carriers of the homozygous GG genotype $(11.9 \pm 4.9$ and $28.6 \pm 6.9 \%$, respectively), however the difference was not statistically significant $(\mathrm{P}=0.563$ and 0.267 , respectively).

The ACS recurrence time (the time from discharge to ACS recurrence) was $17.05 \pm 0.77$ months (95\% CI: 15.54-18.55) for carriers of the homozygous CC genotype of rs1333049, $18.16 \pm 0.56$ months (95\% CI: 17.06-19.26) for carriers of the heterozygous CG genotype and $20.54 \pm 0.75$ months $(95 \% \mathrm{CI}$ : 19.06-22.01) for carriers of the homozygous GG genotype. These differences between carriers of the $C$ allele and homozygous GG individuals were statistically significant (Mantel-Cox log-rank test, $\mathrm{P}=0.004$ and the Wilcoxon signed-rank test, $\mathrm{P}=0.005)$.

The frequencies of recurrent ACS in carriers of different genotypes of $r s 1333049$ depending on the type of hospital care were analyzed (Fig. 5). Group 2 patients who underwent PCI during index hospitalization demonstrated almost equivalent Kaplan-Meier Curves. The survival time (ACS recurrence time) was $18.02 \pm 1.10$ months $(95 \% \mathrm{CI}$ : 17.10-19.99) for patients in group 1 carrying the homozygous CC rs 1333049 polymorphism, 18.54 \pm 0.74 months (95\% CI: 16.86-21.58) for patients with the heterozygous CG genotype and 19.22 \pm 1.21 months (95\% CI: 17.46-19.60) patients with homozygous GG genotype.

The frequency of recurrent ACS and repeated PCI within two years of follow-up differed significantly between group 1 patients with one or two risk C alleles at $r$ s1333049 compared with GG heterozygotes at this locus. The survival time (ACS recurrence time) was 16.22 \pm 1.06 months (95\% CI: 14.14-18.30) in group 2 carriers of the homozygous CC genotype of $r s 1333049,17.73 \pm 0.86$ months $(95 \% \mathrm{CI}$ : 16.05-19.42) for carriers of the heterozygous CG genotype and $21.92 \pm 0.82$ months (95\% CI: 20.31-23.54) in carriers of the homozygous GG genotype. Therefore, the interventional procedure eliminated the presumable CAD-inducing effect of genetic predictors within a relatively short two-year observation period. At the same time, patients who did not receive PCI, had a strong possibility of ACS development. This difference is due to the fact that PCI involves mechanical atherosclerosis burden disruption and stent placement, leading to radical changes the clinical course. 


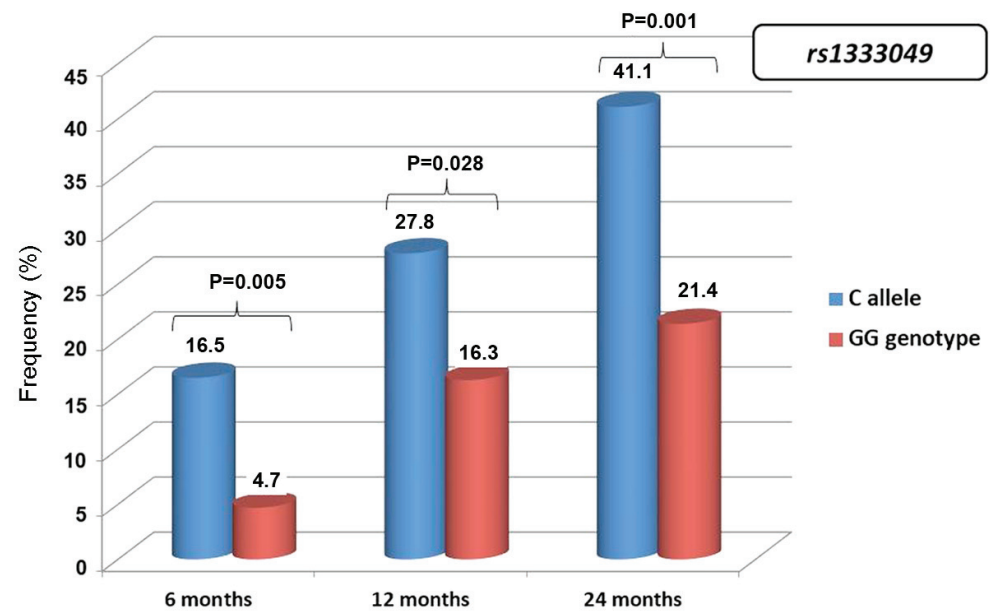

Figure 2. Frequency of recurrent cases of ACS among the carriers of the rs1333049 C allele within the medium-term observation period after MI.

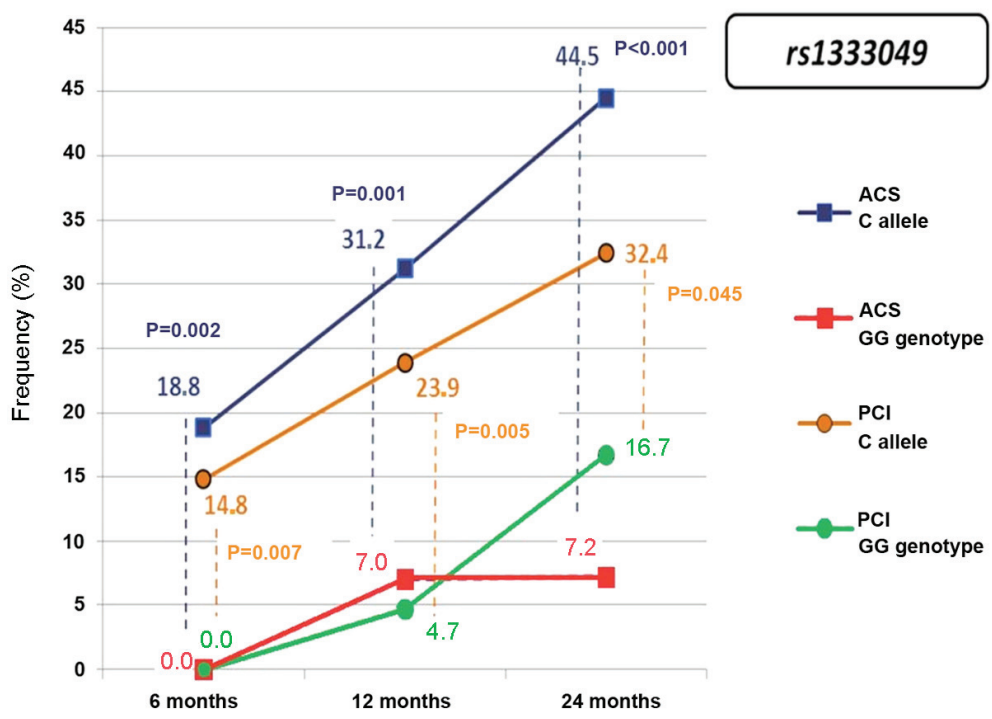

Figure 3. Frequency of recurrent ACS and PCI among the carriers of $r s 1333049$ single nucleotide polymorphism within the follow up period for patients who received a conservative therapy. ACS, acute coronary syndrome; PCI, percutaneous coronary intervention.

\section{Discussion}

The risk factor approach is based on the summing up of the risk factors adverse effects. However, this approach cannot be applied to genetic predictors, since the latter have different genetic mechanisms. Another limiting factor in application of genomic information in risk stratification is high frequency of risk alleles in the population in general (21). Therefore, the use of chromosome 9p21 SNPs in the secondary prevention setting is more reasonable compared with their use in primary prevention.

A larger investigation where the rs $1333049 \mathrm{C}$ risk allele carriership was integrated into the GRACE risk score aimed to assess the combined end point 'cardiac death and recurrent MI' within six months after ACS (13). However, the predictive value of recurrent MI and cardiac death were not statistically significant. From the perspective of clinical medicine, patients with ACS represent a heterogeneous group. In-hospital and post-discharge long-term outcomes depend on the extent of myocardial damage. One of the limitations of the study by Buysschaert et al (13) is the heterogeneity of the cohort of patients with ACS. Specifically, 26.1\% of patients exhibited STEMI, $28.3 \%$ of patients exhibited UA and $41.6 \%$ patients exhibited NSTEMI. Moreover, only half of the enrolled patients underwent surgical intervention: PCI (47.2\%) or coronary artery bypass grafting (3.8\%).

Successful application of genetic determinants as prognostic markers was demonstrated in strictly specific groups of patients. Szpakowicz et al (16) reported a retrospective analysis of 589 patients with STEMI. The rs1333049 and rs 10757278 polymorphic variants of locus 9 p21.3 were significantly associated with overall mortality within 5 years after MI only in high-risk patients according to the GRACE classification (GRACE risk score $\geq 155$ ). Similarly, the Medicine, Angioplasty, or Surgery Study-II, a randomized trial, reported an association between the 9p21.3 locus polymorphism and overall mortality and mortality from cardiac causes in the group of patients with the most severe cases of multi-vessel coronary artery burden (22).

The use of interventional techniques or major surgical procedures may affect disease outcomes for the nearest period 


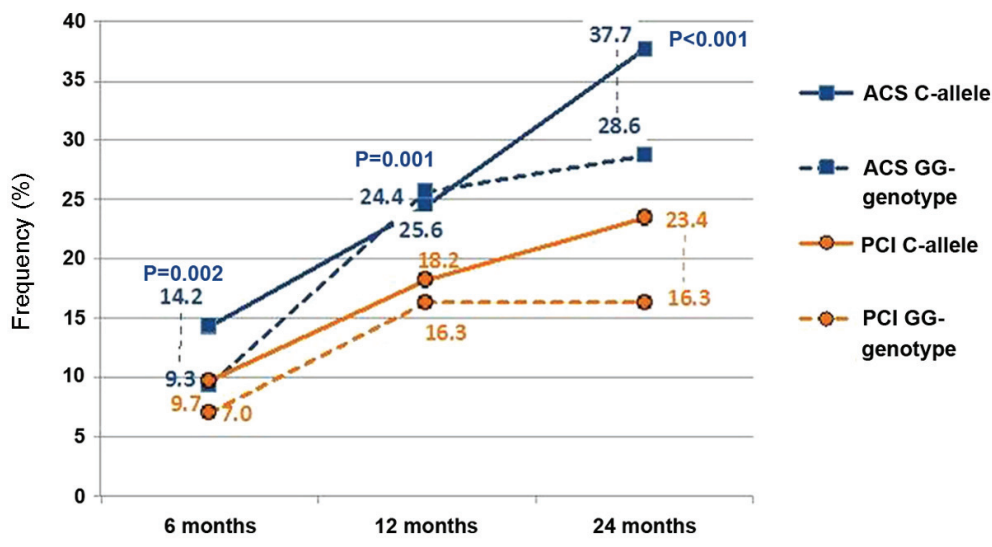

Figure 4. Frequency of recurrent ACS and PCI among the carriers of $r s 1333049$ single nucleotide polymorphism within the follow up period for patients who underwent PCI. ACS, acute coronary syndrome; PCI, percutaneous coronary intervention.

Survivorship function (repeated PCI)

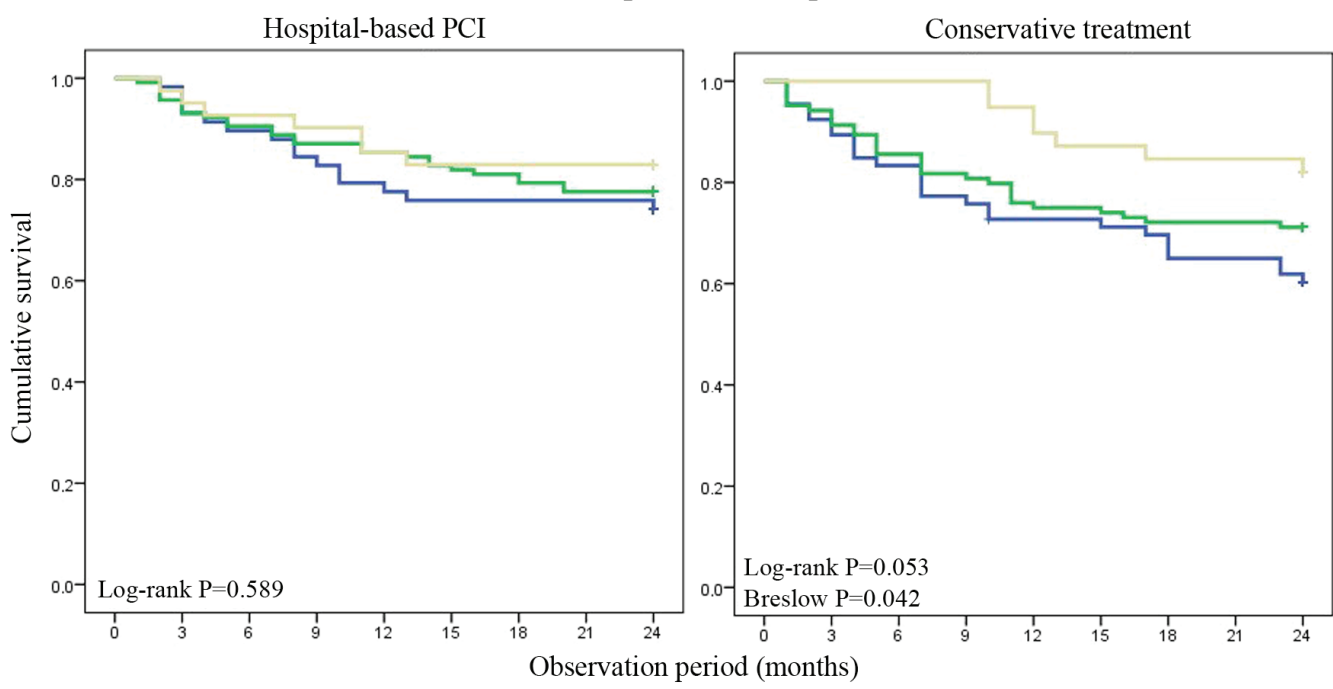

$r s 1333049$

$$
\begin{aligned}
& \sim \text { CC genotype } \\
& \sim \text { CG genotype } \\
& \sim \text { GG genotype } \\
& +\quad \text { cc-censored } \\
& +\quad \text { cg-censored } \\
& +\quad \text { gg-censored }
\end{aligned}
$$

Survivorship function (recurrent ACS)
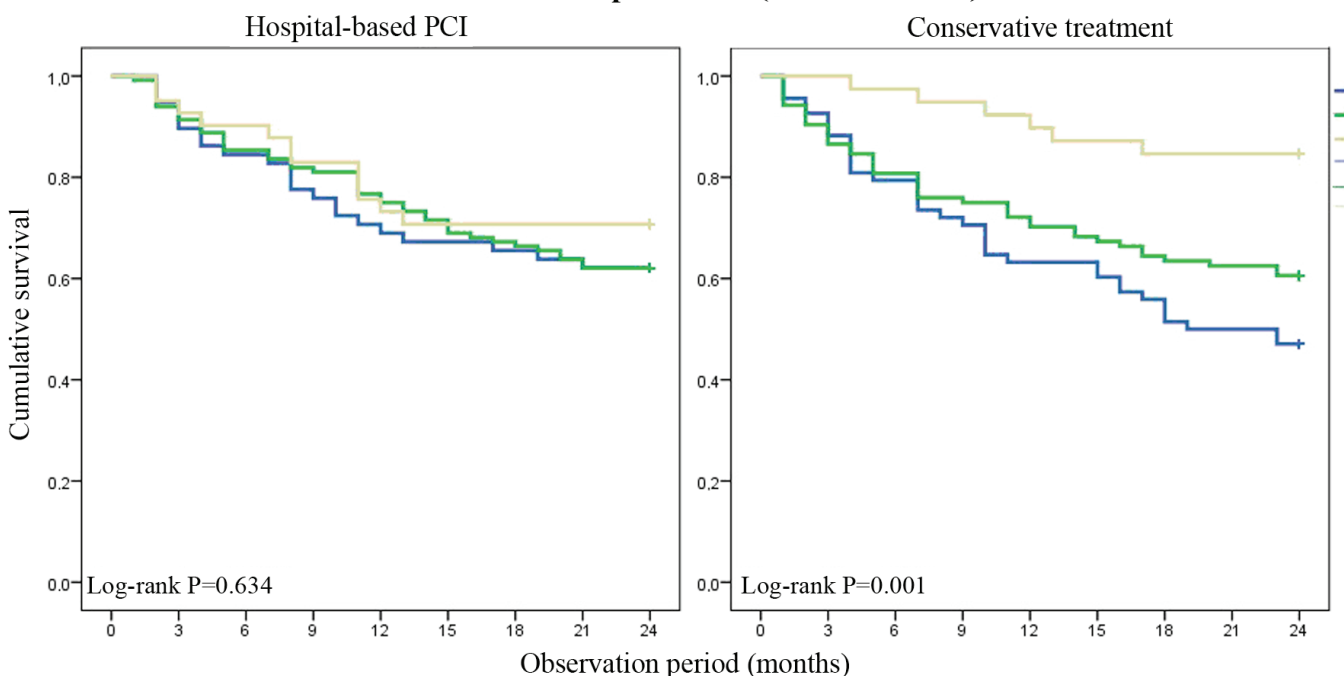

$r s 1333049$

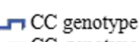

Observation period (months)

Figure 5. Kaplan-Meier Curves of the survival probability for patients receiving conservative treatments and patients who received PCI at index hospitalization with different $r s 1333049$ genotypes during the medium-term observation period after discharge from the hospital.

after MI (20). Previously, no association was revealed between the risk genotypes of $r s 1333040$ and $r s 1333049$ of the 9p21.3 locus and the three-year follow-up outcomes in 2,028 patients with ACS treated with PCI who underwent drug-eluting stent implantation (15). In a large prospective observational study of acute coronary insufficiency (Osaka Acute Coronary 
Insufficiency Study) an association between $r$ s1333049 of the 9p21.3 locus and the risk of recurrent MI was investigated within a year after discharge of 2,022 patients with ACS (23). The authors concluded that there was no association between the risk genotype of rs1333049 and the risk of recurrent $\mathrm{MI}$ in case of PCI, although the $\mathrm{C}$ allele had conferred susceptibility to the first MI (ibid).

In the present study, the correlation coefficient between homozygous CC individuals at rs 1333049 and the GG genotype at $r s 10757278$ amounted to 0.943 . For heterozygous states at both loci the $r_{\mathrm{s}}$ was equal to 0.954 . These findings are consistent with the results of other studies $(3,4,6,7)$ and may be due to these SNPs proximity in the genome.

However, in the logistic regression model, the rs 1333049 SNP exhibited a marginally higher predictive value compared with $r$ 10757278. It should be stated that, to the best of the authors' knowledge, the association between genetic variants and the outcomes for the carriers of the studied genotypes who received conservative treatment for MI has not been previously studied. Moreover, the outcomes for patients (carriers of the studied genotypes with conservative treatment) have also not been compared with the outcomes for patients who underwent PCI at index hospitalization.

The presence or absence of coronary intervention during index hospitalization should be taken into consideration when evaluating genetic markers as predictors of medium-term disease outcomes in patients with MI, since these predictors are relevant only to assess the risk in patients who did not undergo PCI. In the present study, the risk genotype exhibited an independent predictive value in patients with MI who did not undergo revascularization, regardless of the GRACE or TIMI risk scores.

In conclusion, the present pilot study conducted in the Russian Federation, confirmed the results of international genome-wide association studies regarding the association between the 9p21.3 locus SNPs and MI. The results of the present study indicated that the $r$ s 1333049 genotype may be used for medium-term disease outcome prediction in patients with MI who did not undergo coronary intervention at index hospitalization. However, due to a small sample size, the results of the present study may not be generalized to other populations. Therefore, future investigations with larger sample sizes are required to confirm the present results.

\section{Acknowledgements}

The authors are grateful to all colleagues who facilitated the study at the Laboratory of Molecular Genetic Studies of Therapeutic Diseases, Institute of Internal and Preventive Medicine of the Siberian branch of the Russian Academy of Medical Sciences, in Krasnoyarsk Interdistrict Clinical Hospital No. 20 named after I.S. Berzon and in Krasnoyarsk Regional Clinical Hospital.

\section{Funding}

The authors received funding from Professor V.F. VoinoYasenetsky Krasnoyarsk State Medical University. No other specific grants were received from funding agencies in the public, commercial, or not-for-profit sectors.

\section{Availability of data and materials}

The datasets used and/or analyzed during the current study are available from the corresponding author on reasonable request.

\section{Authors' contributions}

SN developed the methodology of the research and was involved in analyzing and interpreting the patient data. IA participated in literature review, was involved in drafting the manuscript, analysing and interpreting the data, and was responsible for final text revision. PS was a major contributor in concept development and literature review, and analyzed and interpreted the patient data and performed the statistical data analysis. OG revised the manuscript, and participated in data collection, literature review and statistical analysis. VM was responsible for carrying out molecular genetic testing (PCR analysis) and DNA extraction. MV was involved in revising the manuscript critically for important intellectual content and made substantial contributions to data analysis. DB participated in source data collection. All authors read and approved the final manuscript.

\section{Ethics approval and consent to participate}

The present study was approved by the Ethics Committee of Professor V.F. Voino-Yasenetsky Krasnoyarsk State Medical University. The patients signed informed consent to participate in the study.

\section{Patient consent for publication}

The authors declare that they obtained informed consent for publication from all the study participants.

\section{Competing interests}

The authors declare that they have no competing interests.

\section{References}

1. Prins BP, Lagou V, Asselbergs FW, Snieder H and Fu J: Genetics of coronary artery disease: Genome-wide association studies and beyond. Atherosclerosis 225: 1-10, 2012.

2. CARDIoGRAMplusC4D Consortium; Deloukas P, Kanoni S, Willenborg C, Farrall M, Assimes TL, Thompson JR, Ingelsson E, Saleheen D, Erdmann J, et al: Large-scale association analysis identifies new risk loci for coronary artery disease. Nat Genet 45: 25-33, 2013.

3. Chan K, Patel RS, Newcombe P, Nelson CP, Qasim A, Epstein SE, Burnett S, Vaccarino VL, Zafari AM, Shah SH, et al: Association between the chromosome 9p21 locus and angiographic coronary artery disease burden: A collaborative meta-analysis. J Am Coll Cardiol 61: 957-970, 2013.

4. Franceschini N, Carty C, Bůžková P, Reiner A, Garrett T, Lin Y, Vöckler JS, Hindorff LA, Cole SA, Boerwinkle E, et al: Association of genetic variants and incident coronary heart disease in multi-ethnic cohorts. The PAGE study. Circ Cardiovase Genet 4: 661-672, 2011.

5. Guo J, Li W, Wu Z, Cheng X, Wang Y and Chen T: Association between 9p21.3 genomic markers and coronary artery disease in East Asians: A meta-analysis involving 9813 cases and 10710 controls. Mol Biol Rep 40: 337-343, 2013. 
6. Horne BD: Chromosome 9p21, risk associations, and biological mechanisms in coronary heart disease. J Am Coll Cardiol 63: 2246-2248, 2014.

7. Munir MS, Wang Z, Alahdab F, Steffen MW, Erwin PJ, Kullo IJ and Murad MH: The association of 9p21-3 locus with coronary atherosclerosis: A systematic review and meta-analysis. BMC Med Genet 6: 66, 2014.

8. Shesternya PA, Shulman VA, Nikulina SYU, Martynova EA, Dyomkina AI, Orlov PS, Maksimov VN and Voyevoda MI Predictive role of chromosome 9p21.3 polymorphisms and their association with family history of coronary heart disease in patients with myocardial infarction. Russian J Cardiol 6: 14-18, 2012 (In Russian).

9. Tajbakhsh A, Khorrami MS, Hassanian SM, Aghasizade M, Pasdar A, Maftouh M, Tabatabai E, Parizadeh SM, Fazeli M, Ferns GA, et al: The 9p21 locus and its potential role in atherosclerosis susceptibility; Molecular mechanisms and clinical implications. Curr Pharm Des 22: 5730-5737, 2016.

10. Ganna A, Magnusson PK, Pedersen NL, de Faire U, Reilly M, Arnöv J, Sundström J, Hamsten A and Ingelsson E: Multilocus genetic risk scores for coronary heart disease prediction. Arterioscler Thromb Vasc Biol 33: 2267-2272, 2013.

11. Paynter NP, Chasman DI, Buring JE, Shiffman D, Cook NR and Ridker PM: Cardiovascular disease risk prediction with or without knowledge of genetic variation at chromosome 9p21.3. Ann Intern Med 150: 65-72, 2009.

12. Ripatti S, Tikkanen E, Orho-Melander M, Havulinna AS, Silander K, Sharma A, Guiducci C, Perola M, Jula A, Sinisalo J, et al: A multilocus genetic risk score for coronary heart disease: Case-control and prospective cohort analyses Lancet 376: 1393-1400, 2010.

13. Buysschaert I, Carruthers KF, Dunbar DR, Peuteman G, Rietzschel E, Belmans A, Hedley A, De Meyer T, Budaj A, Van de Werf F, et al: A variant at chromosome 9p21 is associated with recurrent myocardial infarction and cardiac death after acute coronary syndrome: The GRACE Genetics Study. Eur Heart J 31: 1132-1141, 2010.

14. Adrissino D, Berzuini C, Merlini PA, Mannuccio Mannucci P, Surti A, Burtt N, Voight B, Tubaro M, Peyvandi F, Spreafico M, et al: Influence of 9p21.3 genetic variants on clinical and angiographic outcomes in early-onset myocardial infarction. J Am Coll Cardiol 58: 426-434, 2011.
15. Hoppmann P, Erl A, Türk S, Tiroch K, Mehilli J, Schömig A, Kastrati A and Koch W: No association of chromosome 9 p21.3 variation with clinical and angiographic outcomes after placement of drug-eluting stents. JACC Cardiovasc Interv 2: 1149-1155, 2009.

16. Szpakowicz A, Kiliszek M, Pepinski W, Waszkiewicz E, Franaszczyk M, Skawronska M, Ploski R, NiemcunowiczJanica A, Dobrzycki S, Opolski G, et al: Polymorphism of 9p21.3 locus is associated with 5-year survival in high-risk patients with myocardial infarction. PLoS One 9: e104635, 2014.

17. MONICA Monograph and Multimedia Sourcebook: World's largest study of heart disease, stroke, risk factors, and population trends 1979-2002. Edited by Hugh Tunstall-Pedoe (with 64 other contributors for the WHO MONICA Project). WHO, Geneva, 2003.

18. Malyutina S, Bobak M, Simonova G, Gafarov V, Nikitin Y and Marmot M: Education, marital status, and total and cardiovascular mortality in Novosibirsk, Russia: A prospective cohort study. Ann Epidemiol 14: 244-249, 2004.

19. GRACE Investigators: Rationale and design of the GRACE (Global Registry of Acute Coronary Events) project: A multinational registry of patients hospitalized with acute coronary syndromes. Am Heart J 141: 190-199, 2001.

20. Antman EM, Cohen M, Bernink PJ, McCabe CH, Horacek T, Papuchis G, Mautner B, Corbalan R, Radley D and Braunwald E: The TIMI risk score for unstable angina/non-ST elevation MI: A method for prognostication and therapeutic decision making. JAMA 284: 835-842, 2000

21. Patel RS and Ye S: Genetic determinants of coronary heart disease: New discoveries and insights from genome-wide association study. Heart 97: 1463-1473, 2011.

22. Gioli-Pereira L, Santos PC, Ferreira NE, Hueb WA, Krieger JE and Pereira AC: Higher incidence of death in multi-vessel coronary artery disease patients associated with polymorphisms in chromosome 9p21. BMC Cardiovasc Disord 12: 61, 2012.

23. Hara M, Sakata Y, Nakatani D, Suna S, Usami M, Matsumoto S, Ozaki K, Nishino M, Sato H, Kitamura T, et al: Reduced risk of recurrent myocardial infarction in homozygous carriers of the chromosome 9p21 rs1333049 C risk allele in the contemporary percutaneous coronary intervention era: A prospective observational study. BMJ Open 4: e005438, 2014. 